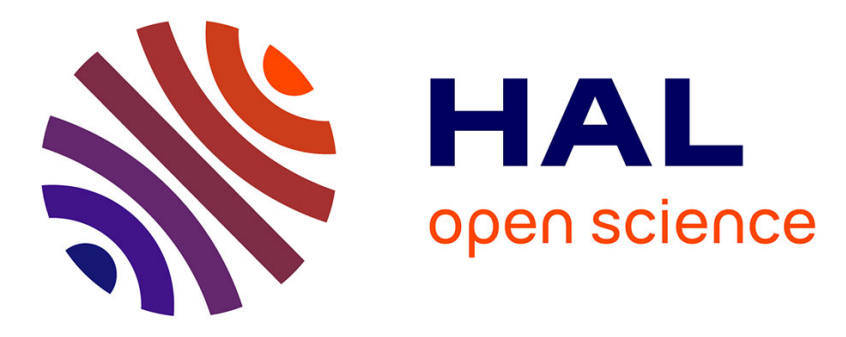

\title{
Transition from quasicrystals to amorphous structures
}

Dariusz Orzechowski, Janusz Wolny

\section{To cite this version:}

Dariusz Orzechowski, Janusz Wolny. Transition from quasicrystals to amorphous structures. Philosophical Magazine, 2005, 86 (03-05), pp.483-487. 10.1080/14786430500251830 . hal-00513566

\section{HAL Id: hal-00513566 https://hal.science/hal-00513566}

Submitted on 1 Sep 2010

HAL is a multi-disciplinary open access archive for the deposit and dissemination of scientific research documents, whether they are published or not. The documents may come from teaching and research institutions in France or abroad, or from public or private research centers.
L'archive ouverte pluridisciplinaire HAL, est destinée au dépôt et à la diffusion de documents scientifiques de niveau recherche, publiés ou non, émanant des établissements d'enseignement et de recherche français ou étrangers, des laboratoires publics ou privés. 


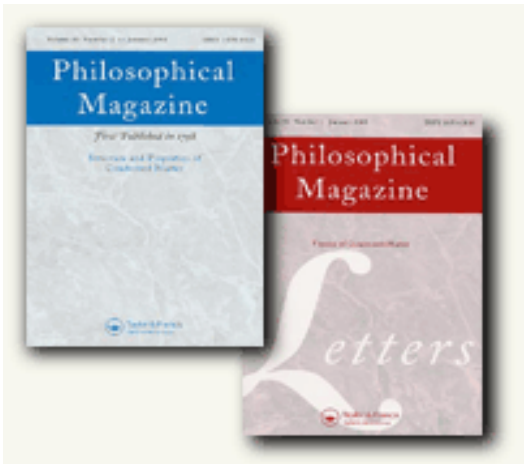

\section{Transition from quasicrystals to amorphous structures}

\begin{tabular}{|c|c|}
\hline Journal: & Philosophical Magazine \& Philosophical Magazine Letters \\
\hline Manuscript ID: & TPHM-05-May-0227.R1 \\
\hline Journal Selection: & Philosophical Magazine \\
\hline $\begin{array}{r}\text { Date Submitted by the } \\
\text { Author: }\end{array}$ & 07-Jul-2005 \\
\hline Complete List of Authors: & $\begin{array}{l}\text { Orzechowski, Dariusz; AGH University of Science and Technology, } \\
\text { Faculty of Physics and Applied Computer Science } \\
\text { Wolny, Janusz; AGH University of Science and Technology, Faculty } \\
\text { of Physics and Applied Computer Science }\end{array}$ \\
\hline Keywords: & quasicrystals, disordered materials, diffraction \\
\hline Keywords (user supplied): & \\
\hline
\end{tabular}

\section{今 ScholarONE" \\ Manuscript Central}




\title{
Transition from quasicrystals to amorphous structures
}

\author{
D. ORZECHOWSKI and J. WOLNY
Faculty of Physics and Applied Computer Science, AGH University of Science and Technology, al. Mickiewicza 30, 30-059 Krakow, POLAND

\begin{abstract}
For the model structure the smooth transition from perfect quasicrystalline to amorphous structure has been observed and changes in the diffraction patterns has been investigated. It turned out that all dependencies can be easily handled by means of some well-defined probablility distributions. Given these distributions one can perform an appropriate calculations in the average unit cell method and describe the transition in a simple and consistent way.
\end{abstract}

Keywords: Quasicrystals; Diffraction; Disordered materials

\section{Introduction}

The diffraction pattern of any structure can be obtained by the Fourier transformation of its atomic positions. Classical crystallographic approach, however is based on the concept of unit cell and reciprocal lattice, therefore its validity is constrained to the analysis of perfectly periodic crystals. If a given structure is not of this type, the unit cell doesn't exist and this approach fails. It has been shown [1] that diffraction pattern of such structures may be correctly described in the average unit cell approach based on the reference lattice concept. In this approach periodicity of real structure is not important, nevertheless when it holds, the average unit cell is fully equivalent to the classic unit cell. It is possible to construct probability distributions of positions of atoms (nodes) relative to the unit cell and then calculate Fourier transformation to get so-called envelope function correctly describing intensities for certain well-defined scattering vectors.

The diffraction pattern of perfect crystals consists of Bragg peaks corresponding to the points of the appropriate reciprocal lattice. Unfortunately, for all non-periodic even well-defined structures the classic crystallographic concepts of unit cell and reciprocal lattice are nearly useless. Although the diffraction pattern of perfect quasicrystals also consists of Bragg peaks, the reciprocal lattice of such structure does not exist. As an example, in this work Fibonacci chain and its modifications has been investigated.

Fibonacci chain is well-known and thoroughly analysed one-dimensional model of perfect quasicrystal [2]. It can be obtained for example by iterative substituting $L \rightarrow L S$ and $S \rightarrow L$, where $S$ is the shorter distance between the nodes of the Fibonacci chain and $L$ is $\tau$ times longer (golden number $\tau=(1+\sqrt{5}) / 2 \approx 1.618$. In this work $S$ has been taken as the unity length. Another popular method of constructing the Fibonacci chain is the projection of the strip of two-dimensional square lattice onto certain well-defined direction.

The diffraction pattern of Fibonacci chain consists of main peaks and series of satellite peaks. We can construct the average unit cell of the length $a$ equal to the average distance between nodes (given by $a=1+1 / \tau^{2} \approx 1.382$ ) and obtain probability distribution $P(u)$ in such average unit cell, where $u$ is the shortest distance between positions of nodes of Fibonacci chain and nodes of the periodic reference lattice with period equal to $a$. Fourier transformation of such probability 
distribution gives an envelope function which shows correct intensities of all main reflections. By creating separate average unit cell of the bigger length $b=a \cdot \tau$ and appropriate $P(v)$ distributions we are able to describe correctly also intensities of all satellite peaks. In the $(u, v)$ parameter space probability distribution $P(u, v)$ proves to be only one line with the constant value on it and zero elsewhere (fig.1).

[Insert figure 1 about here]

Exactly the same reasoning can be used to describe disordered structures. For example, the perfect Fibonacci chain nodes positions $F_{n}$ (where $n$ is a node number) can be perturbed by adding to them a random shift $\varepsilon$ of the Gaussian distribution with the standard deviation $\sigma$. So, the new position of the $n$-th node can be written as follows: $f_{n}=F_{n}+\varepsilon$. Small sigma parameter (relative to the length of the average unit cell) in periodic systems leads to the well-known Debye-Waller factor describing the thermal motions of the atoms.

The scope of the work was to observe influence of the $\varepsilon$. value on the diffraction pattern. Changes in the $P(u, v)$ distributions as a function of the $\sigma$ parameter also has been investigated. The fact that there are two well-defined extreme cases: for $\sigma=0$ we have just pure Fibonacci chain (i.e. perfect one-dimensional quasicrystal) and for $\sigma \rightarrow \infty$ the structure becomes completely random, proved to be very helpful and allowed to control validity of calculations. Between these two extremes, however, exist large variety of partially disordered strucures and we can observe transition from amorphous to the quasicrystalline state.

Two distinct cases have been taken into consideration. First, the $\sigma$ parameter has been set to constant value throughout the whole structure, that is $\sigma$ has been independent of node number $n$. In the second case $\sigma$ has been set to be linearly rising function of $n$. As the more intersting results has been obtained in the second case, only this case will be described below.

\section{The description of the model}

The positions of the nodes of pure Fibonacci chain have been perturbed by adding to them a random value $\varepsilon$ of the Gaussian distribution with the standard deviation $\sigma$. But, $\sigma$ parameter has been set to be linear function of node number $n$, that is $\sigma(n)=\alpha \cdot n$, where $\alpha$ is arbitrary chosen positive constant. Such dependency preserves short range order but makes the correlations vanish with distance. What is very important, this dependency $(\sigma(n))$ has been taken into consideration only inside clusters of relatively small size $N$ (varied from about 5 to 200), and not in the entire, virtually infinite chain. These clusters have been taken as a parts of Fibonacci chain modified in the way described above. The calculations have been performed for a big number of separate clusters and then average values have been obtained. This procedure helped to get rid of fluctuations coming from random factors as well as relatively small cluster sizes. One should also note that cluster size $N$ has not been fixed to be constant but could fluctuate around chosen value in such a way that average $N$ has been preserved.

First, the diffraction patterns for various cluster sizes and various $\alpha$ parameters has been calculated. One can observe two distinct parts of the diffraction patterns: first consists of quite sharp Bragg peaks (the width of these peaks reduces as the cluster size grows) and continuous part which is constant if intensities are scaled by $N$ and not $N^{2}$ as usual (fig.2).

[Insert figure 2 about here] 
The intensity of the first main Bragg peak $(k \approx 4.546)$ versus cluster size has been investigated (fig.3). It's clearly visible that for large $N$ Bragg peaks vanish and belong to the continuous part of the diffraction pattern.

[Insert figure 3 about here]

Behaviour of probablility distribution $P(u, v)$ as well as its projections onto different directions has been observed. For pure Fibonacci chain $u(v)$ is just a straight line with constant probablility on it and zero elsewhere. This line turns out to diffuse in both directions - quite slowly along the line and much faster in the perpendicular direction (fig.4).

\section{[Insert figure 4 about here]}

Projection onto $u$ axis gives probablility distribution $P(u)$ which is strictly correlated with the main peaks intensities. The Fourier transformation of $P(u)$ distribution gives an envelope function correctly describing intensities for all scattering vectors (or, in our one-dimensional case just numbers) $k=n k_{0}$, where $n=1,2,3, \ldots$ is a natural number and $k_{0}=2 \pi / a$ is a basic scattering vector (number) related with average distance between nodes of pure Fibonacci chain $a$. For small cluster sizes and small $\alpha$ parameters, $P(u)$ is close to rectangular distributions as in pure Fibonacci chain. For bigger $N$, however, $P(u)$ distributions become more flat and have nonzero value for the whole $u$ range (fig.5)

[Insert figure 5 about here]

\section{Conclusions}

In this model for the first time a smooth transition from perfect quasicrystal to the amorphous state has been observed. In such a simple model one can easily investigate the behaviour of the diffraction pattern depending on such things as a cluster size and its dispersion and parameter $\alpha$ simulating vanishing correlations of atomic positions with regard to the perfect quasicrystalline structure. All dependencies can be handled in simple way by means of probablility distribution $P(u, v)$ and its projections onto certain directions. Given probability distributions one can quickly perform all appropriate calculations in the average unit cell method and this way one can describe transition from quasicrystalline to amorphous state in consistent and well-defined way.

\section{References}

[1] J. Wolny, The reference lattice concept and its application to the analysis of diffraction patterns, Philosophical Magazine, A77, (1998), 395-412

[2] M. Senechal, Quasicrystals and geometry, University Press Cambridge, (1995) 


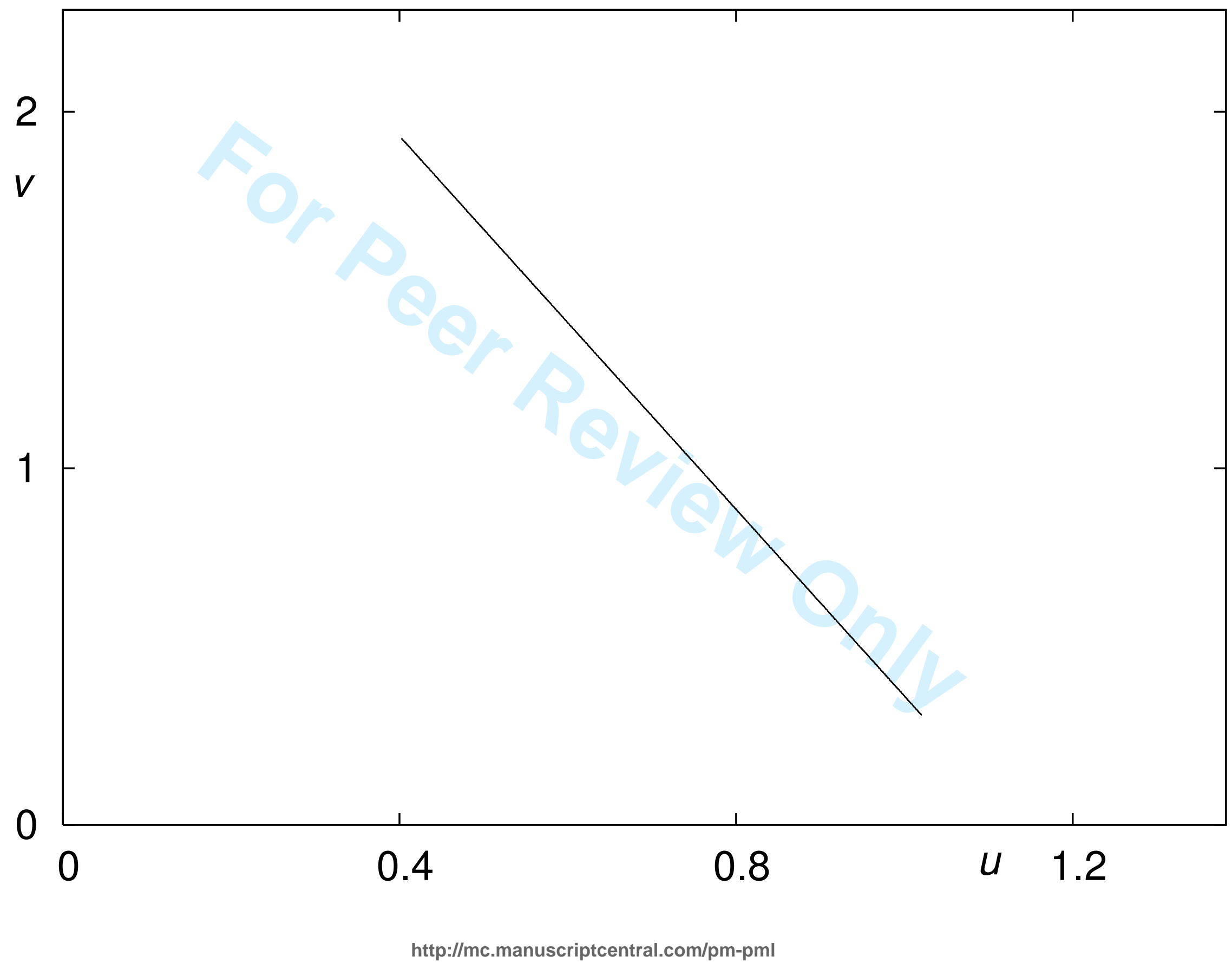




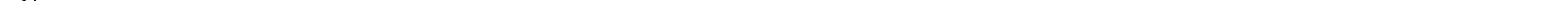




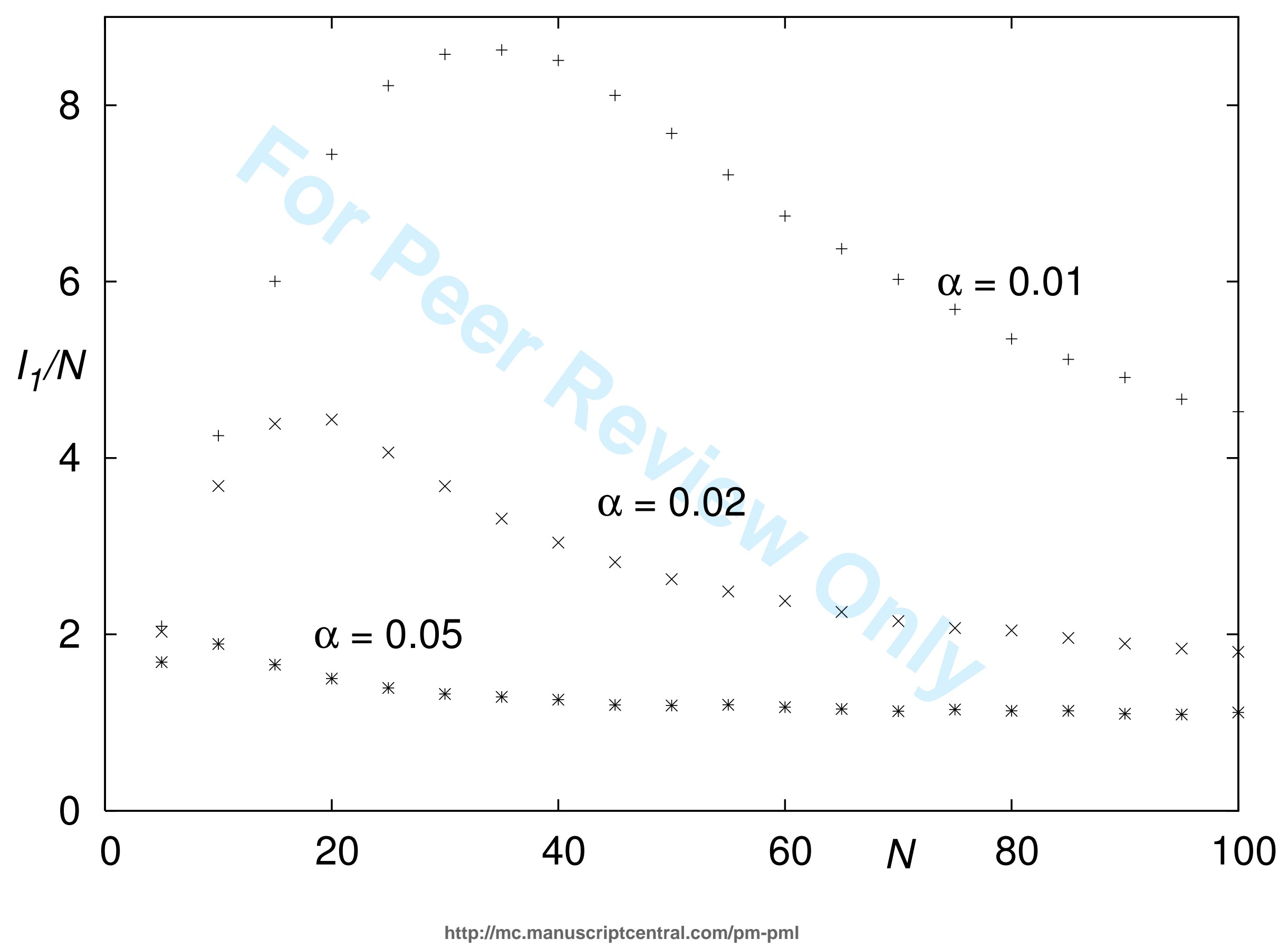




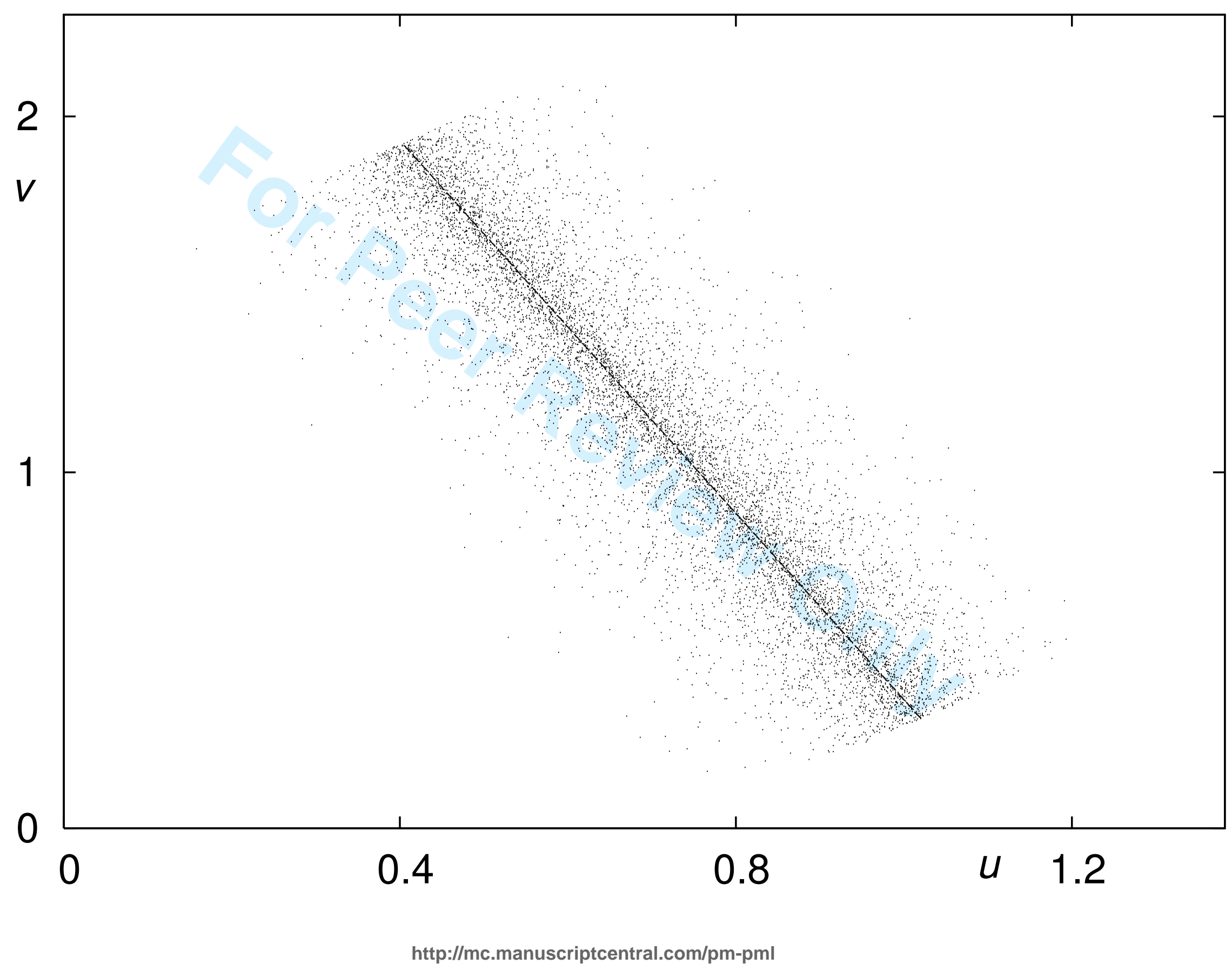




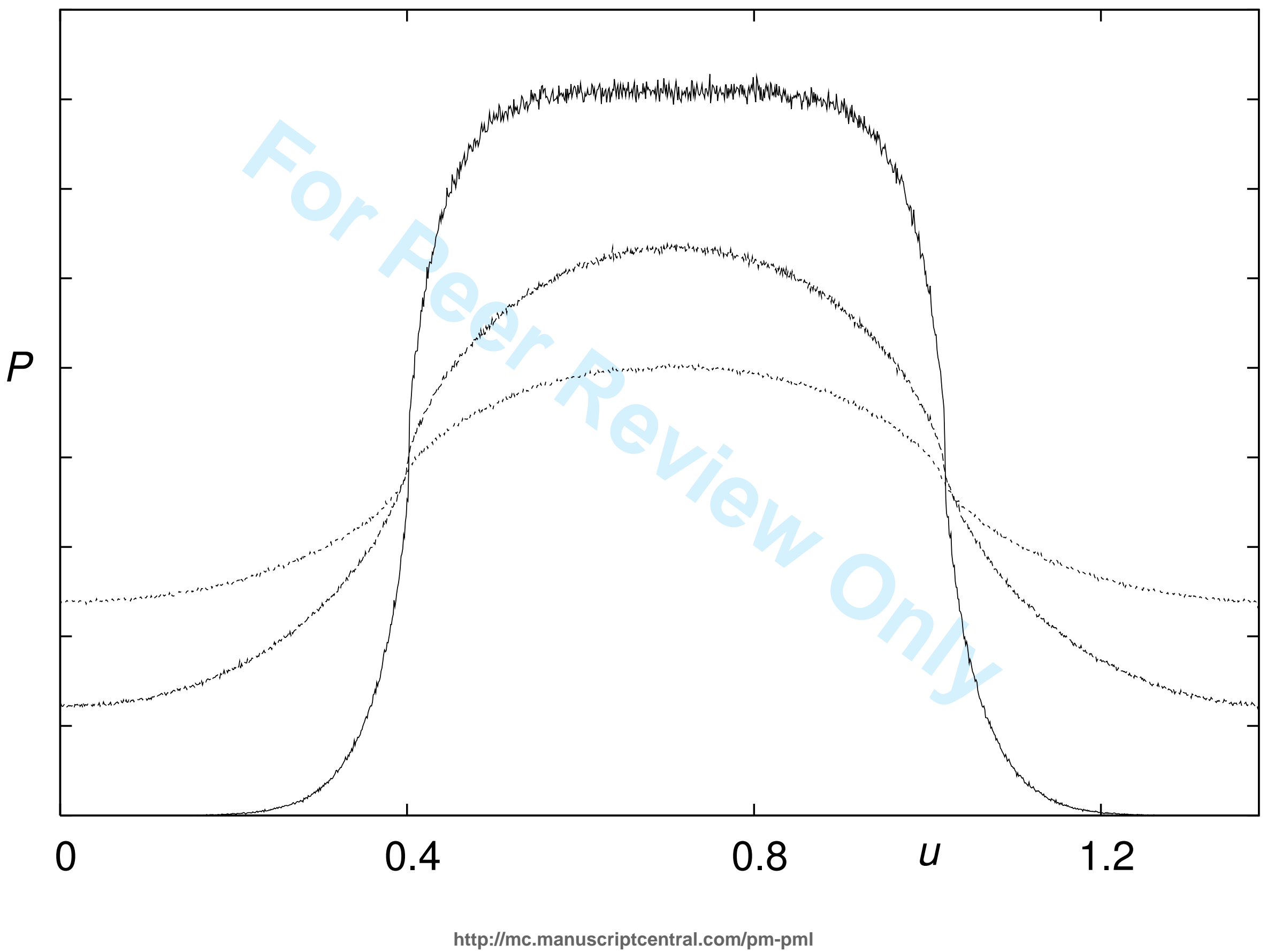

